Supporting Information to

\title{
Energetic Stabilization of Carboxylic Acid Conformers by Manganese Atoms and Clusters
}

Sandra M. Lang, ${ }^{1}$ Thorsten M. Bernhardt, ${ }^{1}$ Joost M. Bakker, ${ }^{2}$ Robert N. Barnett, ${ }^{3}$ Uzi

Landman ${ }^{3}$

${ }^{1}$ Institute of Surface Chemistry and Catalysis, University of Ulm, Albert-Einstein-Allee 47, 89069 Ulm, Germany

${ }^{2}$ Radboud University, Institute for Molecules and Materials, FELIX Laboratory,

Toernooiveld 7, 6525 ED Nijmegen, The Netherlands

${ }^{3}$ School of Physics, Georgia Institute of Technology, Atlanta, GA 30332-0430, USA

\section{Comparison of Theoretical Methods}

Table S1: Calculated geometric parameters of free acetic acid (AA) in trans- and cis-geometry obtained with the software package VASP ${ }^{1-4}$ as well as BO-SDFT-MD. ${ }^{5}$ Bond lenght are given in $\AA$, frequencies in $\mathrm{cm}^{-1}$

\begin{tabular}{|c|c|c|c|c|}
\hline & \multicolumn{2}{|c|}{$\operatorname{trans}-\mathbf{A A}$} & \multicolumn{2}{|c|}{ cis-AA } \\
\hline & VASP & BO-SDFT-MD & VASP & BO-SDFT-MD \\
\hline $\mathrm{C}=\mathrm{O}$ & 1.219 & 1.221 & 1.212 & 1.213 \\
\hline $\mathrm{C}-\mathrm{O}$ & 1.369 & 1.383 & 1.375 & 1.389 \\
\hline $\mathrm{C}-\mathrm{C}$ & 1.503 & 1.505 & 1.513 & 1.515 \\
\hline $\mathrm{C}-\mathrm{H}$ & $1.094 / 1.099 / 1.100$ & $1.092 / 1.096 / 1.097$ & $1.095 / 1.101 / 1.101$ & $1.091 / 1.098 / 1.099$ \\
\hline $\mathrm{O}-\mathrm{H}$ & 0.981 & 0.980 & 0.977 & 0.976 \\
\hline $\mathrm{OCO}$ & $122.3^{\circ}$ & $122.0^{\circ}$ & $119.4^{\circ}$ & $119.8^{\circ}$ \\
\hline $\mathrm{CC}-\mathrm{O}$ & $111.5^{\circ}$ & $111.5^{\circ}$ & $115.4^{\circ}$ & $114.0^{\circ}$ \\
\hline $\mathrm{CC}=\mathrm{O}$ & $126.3^{\circ}$ & $126.5^{\circ}$ & 125.2 & $126.1^{\circ}$ \\
\hline $\mathrm{COH}$ & $105.9^{\circ}$ & $105.2^{\circ}$ & $110.0^{\circ}$ & $109.04^{\circ}$ \\
\hline$v(\mathrm{C}=\mathrm{O})$ & 1761 & 1721 & 1797 & 1762 \\
\hline
\end{tabular}


Table S2: Calculated geometric parameters of $\mathrm{Mn}(\mathrm{AA})^{+}$in trans- and cis-geometry obtained with the software package VASP ${ }^{1-4}$ as well as BO-SDFT-MD. ${ }^{5}$ Bond lengths are given in $\AA$, frequencies in $\mathrm{cm}^{-}$ ${ }^{1}$. It should be noted that VASP consistently predicts a stronger Mn-AA interaction and a shorter Mn-O bond length, whereas all other geometric parameters are similar for both methods.

\begin{tabular}{l|l|l|l|l} 
& \multicolumn{2}{|c|}{ Mn(trans-AA) } & \multicolumn{2}{c}{ Mn(cis-AA) $^{+}$} \\
& \multicolumn{1}{|c|}{ VASP } & \multicolumn{1}{|c}{ BO-SDFT-MD } & \multicolumn{1}{|c}{ VASP } & \multicolumn{1}{c}{ BO-SDFT-MD } \\
\hline $\mathrm{Mn}-\mathrm{O}$ & 2.040 & 2.223 & 2.063 & 2.199 \\
$\mathrm{C}=\mathrm{O}$ & 1.260 & 1.257 & 1.252 & 1.248 \\
$\mathrm{C}-\mathrm{O}$ & 1.318 & 1.332 & 1.328 & 1.341 \\
$\mathrm{C}-\mathrm{C}$ & 1.485 & 1.491 & 1.488 & 1.495 \\
$\mathrm{C}-\mathrm{H}$ & $1.094 / 1.101 / 1.101$ & $1.092 / 1.097 / 1.098$ & $1.094 / 1.102 / 1.102$ & $1.092 / 1.098 / 1.099$ \\
$\mathrm{O}-\mathrm{H}$ & 0.985 & 0.983 & 0.981 & 0.980 \\
$\mathrm{OCO}$ & $120.3^{\circ}$ & $120.9^{\circ}$ & $116.9^{\circ}$ & $117.6^{\circ}$ \\
$\mathrm{CC}-\mathrm{O}$ & $114.5^{\circ}$ & $113.7^{\circ}$ & $120.2^{\circ}$ & $119.2^{\circ}$ \\
$\mathrm{CC}=\mathrm{O}$ & $125.2^{\circ}$ & $125.4^{\circ}$ & $122.8^{\circ}$ & $123.1^{\circ}$ \\
$\mathrm{COH}$ & $11.1^{\circ}$ & $110.1^{\circ}$ & $112.5^{\circ}$ & $111.3^{\circ}$ \\
$\mathrm{v}(\mathrm{C}=\mathrm{O})$ & 1598 & 1599 & 1646 & 1648
\end{tabular}




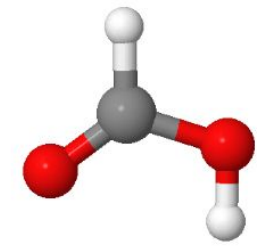

trans-FA

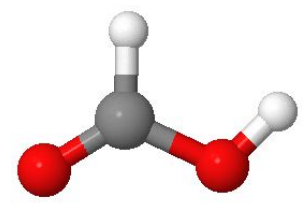

cis-FA experiment: $\Delta \mathrm{E}_{\mathrm{tc}}=0.169 \pm 0.004 \mathrm{eV}^{6}$

theory (VASP, this work): $\Delta \mathrm{E}_{\mathrm{tc}}=0.16 \mathrm{eV}$

Table S3: Calculated geometric parameters of free formic acid in trans- and cis-geometry in comparison with experimental values. All values are given in $\AA$, except otherwise specified.

\begin{tabular}{|c|c|c|c|c|c|c|c|}
\hline & \multicolumn{5}{|c|}{ trans-FA } & \multicolumn{2}{|r|}{ cis-FA } \\
\hline & VASP & Kwei $^{7}$ & Schomaker $^{8}$ & Karle $^{9}$ & Lerner $^{10}$ & VASP & Bjarnov $^{11}$ \\
\hline $\mathrm{C}=\mathrm{O}$ & 1.213 & $1.2025 \pm 0.010$ & $1.213 \pm 0.026$ & $1.23 \pm 0.010$ & $1.245 \pm 0.002$ & 1.206 & $1.1945 \pm 0.0031$ \\
\hline $\mathrm{C}-\mathrm{O}$ & 1.356 & $1.343 \pm 0.010$ & $1.368 \pm 0.034$ & $1.36 \pm 0.01$ & $1.312 \pm 0.002$ & 1.363 & $1.3520 \pm 0.0028$ \\
\hline $\mathrm{C}-\mathrm{H}$ & 1.107 & $1.097 \pm 0.005$ & 1.09 & 1.09 & $1.085 \pm 0.02$ & 1.114 & $1.1050 \pm 0.0043$ \\
\hline $\mathrm{O}-\mathrm{H}$ & 0.983 & $0.972 \pm 0.005$ & $0.97 \pm 0.05$ & $0.97 \pm 0.05$ & $0.95 \pm 0.02$ & 0.978 & $0.9555 \pm 0.0053$ \\
\hline $\mathrm{OCO}$ & $125.2^{\circ}$ & $124.9^{\circ} \pm 1^{\circ}$ & $123.5^{\circ} \pm 3^{\circ}$ & $122.4^{\circ} \pm 1^{\circ}$ & $124.3^{\circ} \pm 10^{\circ}$ & $122.2^{\circ}$ & $122.12^{\circ} \pm 0.37^{\circ}$ \\
\hline $\mathrm{HC}-\mathrm{O}$ & $109.4^{\circ}$ & & & & & $113.5^{\circ}$ & $114.64^{\circ} \pm 0.56^{\circ}$ \\
\hline $\mathrm{HC}=\mathrm{O}$ & $125.5^{\circ}$ & $124.1^{\circ} \pm 2^{\circ}$ & & & & $124.3^{\circ}$ & $123.23^{\circ} \pm 0.58^{\circ}$ \\
\hline $\mathrm{COH}$ & $106.9^{\circ}$ & $106.3^{\circ} \pm 1^{\circ}$ & & 105 & $107.8^{\circ} \pm 1^{\circ}$ & $109.4^{\circ}$ & $109.68^{\circ} \pm 0.44^{\circ}$ \\
\hline
\end{tabular}


Table S4: Calculated vibrational frequencies of free trans- and cis-formic acid in comparison with experimental values.

\begin{tabular}{l|l|l|l|l} 
& \multicolumn{2}{|c|}{ trans-FA } & \multicolumn{2}{c}{ cis-FA } \\
\hline \multicolumn{1}{c|}{ Mode $^{(\mathrm{a})}$} & Gas Phase $^{12}$ & \multicolumn{1}{c}{ VASP } & Solid Ar ${ }^{13}$ & VASP \\
\hline$\nu \mathrm{C}=\mathrm{O}$ & 1770 & 1762 & $1808 / 1807$ & 1808 \\
$\delta \mathrm{CH}$ & 1387 & 1349 & 1392 & 1362 \\
$\delta \mathrm{OH}$ & 1229 & $1254^{(\mathrm{b})}$ & $1243 / 1249$ & 1220 \\
$\nu \mathrm{C}-\mathrm{O}$ & 1105 & $1075^{(\mathrm{b})}$ & $1107 / 1105$ & 1077 \\
$\delta \mathrm{CH}$ & 1033 & 996 & - & 980 \\
$\tau \mathrm{OH}$ & 638 & 673 & $503 / 505$ & 554 \\
OCO def & 625 & 602 & 662 & 633
\end{tabular}

(a) mode assignment according to Ref. ${ }^{12}$ ( $v$ : stretch, $\delta$ : bending, $\tau$ : torsion, def: deformation)

(b) both modes have contributions from $\delta \mathrm{OH}$ and $v \mathrm{C}-\mathrm{O}$

\subsection{Energies, Geometric Parameters, and Vibrational Frequencies of Acetic Acid (AA)}

\section{Conformers}

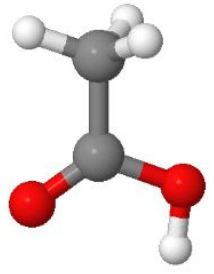

trans-AA

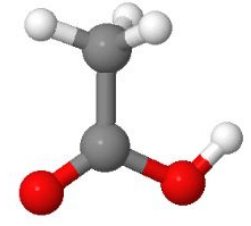

cis-AA experiment: data not available

theory (VASP, this work): $\Delta \mathrm{E}_{\mathrm{tc}}=0.21 \mathrm{eV}$

theory (other work): $\Delta \mathrm{E}_{\mathrm{tc}}=0.30 \mathrm{eV},{ }^{14} 0.22$ $\mathrm{eV},{ }^{15} 0.22 \mathrm{eV},{ }^{16} 0.23 \mathrm{eV}^{17}$

Table S5: Calculated geometric parameters of free acetic acid in trans- and cis-geometry in comparison with experimental values. All values are given in $\AA$, except otherwise specified.

\begin{tabular}{|c|c|c|c|c|c|}
\hline & \multicolumn{3}{|c|}{$\operatorname{trans}-\mathbf{A A}$} & \multicolumn{2}{|c|}{ cis-AA } \\
\hline & VASP & Hellwege $^{18}$ & Derissen $^{19}$ & VASP & \\
\hline $\mathrm{C}=\mathrm{O}$ & 1.219 & 1.212 & $1.214 \pm 0.003$ & 1.212 & no \\
\hline $\mathrm{C}-\mathrm{O}$ & 1.369 & 1.361 & $1.364 \pm 0.003$ & 1.375 & experimental data \\
\hline $\mathrm{C}-\mathrm{C}$ & 1.503 & 1.517 & $1.520 \pm 0.005$ & 1.513 & available \\
\hline $\mathrm{C}-\mathrm{H}$ & $1.094 / 1.099 / 1.100$ & 1.100 & $1.102 \pm 0.010$ & $1.095 / 1.101 / 1.101$ & \\
\hline $\mathrm{O}-\mathrm{H}$ & 0.981 & & 0.97 & 0.977 & \\
\hline $\mathrm{OCO}$ & $122.3^{\circ}$ & $123.0^{\circ}$ & $122.5^{\circ}$ & $119.4^{\circ}$ & \\
\hline $\mathrm{CC}-\mathrm{O}$ & $111.5^{\circ}$ & $110.6^{\circ}$ & $110.6^{\circ} \pm 0.6^{\circ}$ & $115.4^{\circ}$ & \\
\hline $\mathrm{CC}=\mathrm{O}$ & $126.3^{\circ}$ & $126.6^{\circ}$ & $126.6^{\circ} \pm 0.6^{\circ}$ & & \\
\hline $\mathrm{COH}$ & $105.9^{\circ}$ & & $107.0^{\circ}$ & $110.0^{\circ}$ & \\
\hline
\end{tabular}


Table S6: Calculated vibrational frequencies of free trans- and cis-acetic acid in comparison with experimental values.

\begin{tabular}{l|l|l|l|l} 
& \multicolumn{2}{|c|}{ trans-AA } & \multicolumn{2}{c}{ cis-AA } \\
\hline \multicolumn{1}{c|}{ Mode $^{(a)}$} & \multicolumn{1}{|c|}{ Gas Phase $^{12}$} & \multicolumn{1}{|c}{ VASP } & \multicolumn{1}{c}{ Solid Ar ${ }^{20}$} & \multicolumn{1}{c}{ VASP } \\
\hline$\nu \mathrm{C}=\mathrm{O}$ & 1788 & 1761 & 1807 & 1797 \\
$\mathrm{CH}_{3}$ def & $1430 / 1430 / 1382$ & $1426 / 1420 / 1354$ & $1448 / 1445 / 1368$ & $1438 / 1423 / 1340$ \\
$\delta \mathrm{OH}$ & 1264 & $1290^{(\mathrm{b})}$ & 1272 & 1240 \\
$\nu \mathrm{C}-\mathrm{O}$ & 1182 & $1152^{(\mathrm{b})}$ & 1193 & 1159 \\
$\gamma \mathrm{CH}_{3}$ & $1048 / 989$ & $1020 / 957$ & $1042 / 982$ & $1015 / 951$ \\
$\nu \mathrm{C}-\mathrm{C}$ & 847 & 842 & 849 & 834 \\
$\mathrm{OCO}$ def & 657 & 559 & 458 & 574 \\
$\delta \mathrm{C}=\mathrm{O}_{\text {op }}$ & 642 & $654^{(\mathrm{c})}$ & & $579^{(\mathrm{e})}$ \\
$\mathrm{CCO}$ def & 581 & 408 & & 418 \\
$\tau \mathrm{C}-\mathrm{O}$ & 534 & $530^{(\mathrm{c})}$ & & $488^{(\mathrm{e})}$ \\
$\tau \mathrm{CH} \mathrm{H}_{3}$ & 93 & 33 & & 121
\end{tabular}

(a) mode assignment according to Ref. ${ }^{12}$ ( $v$ : stretch, $\delta$ : bending, $\tau$ : torsion, def: deformation)

(b) both modes have contributions from $\delta \mathrm{OH}$ and $v \mathrm{C}-\mathrm{O}$

(c) both modes have contributions from $\delta \mathrm{C}=\mathrm{O}_{\mathrm{op}}$ and $\tau \mathrm{C}-\mathrm{O}$

\subsection{Energies, Geometric Parameters, and Vibrational Frequencies of Methyl Acetate} (MA) Conformers

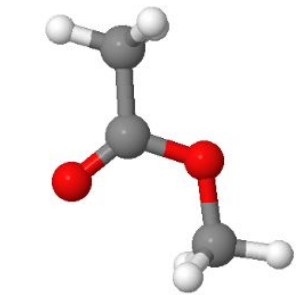

trans-MA

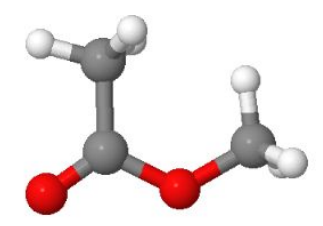

experiment: $\Delta \mathrm{E}_{\mathrm{tc}}=0.37 \pm 0.04 \mathrm{eV}^{21}$

theory (VASP, this work): $\Delta \mathrm{E}_{\mathrm{tc}}=0.34 \mathrm{eV}$

Table S7: Calculated geometric parameters of free methyl acetate in trans- and cis-geometry. All values are given in $\AA$, except otherwise specified

\begin{tabular}{l|l|l|l|l} 
& \multicolumn{2}{|c|}{ trans-MA } & \multicolumn{2}{c}{ cis-MA } \\
\hline & \multicolumn{1}{|c|}{ VASP } & & \multicolumn{1}{|c}{ VASP } & \\
\hline $\mathrm{C}=\mathrm{O}$ & 1.220 & no & 1.216 & no \\
$\mathrm{C}-\mathrm{O}$ & 1.364 & experimental data & 1.369 & experimental data \\
$\mathrm{O}-\mathrm{CH}$ & 1.444 & available & 1.437 & available \\
$\mathrm{C}-\mathrm{C}$ & 1.505 & & 1.513 & \\
$\mathrm{C}-\mathrm{H}(\mathrm{O})$ & $1.099 / 1.099 / 1.095$ & & $1.099 / 1.099 / 1.097$ & \\
$\mathrm{C}-\mathrm{H}(\mathrm{C})$ & $1.100 / 1.099 / 1.095$ & & $1.100 / 1.100 / 1.094$ & \\
$\mathrm{OCO}$ & $123.3^{\circ}$ & $117.9^{\circ}$ & \\
$\mathrm{CC}-\mathrm{O}$ & $110.8^{\circ}$ & & $118.9^{\circ}$ & \\
$\mathrm{CC}=\mathrm{O}$ & $125.9^{\circ}$ & & $123.3^{\circ}$ & \\
$\mathrm{COC}$ & $115.0^{\circ}$ & & $123.0^{\circ}$ &
\end{tabular}


Table S8: Calculated vibrational frequencies of free trans- and cis-methyl acetate in comparison with experimental values.

\begin{tabular}{|c|c|c|c|c|}
\hline \multirow[b]{2}{*}{ Mode $^{(a)}$} & \multicolumn{2}{|c|}{$\operatorname{trans-MA}$} & \multicolumn{2}{|c|}{ cis-MA } \\
\hline & Gas Phase $^{12}$ & VASP & Solid Ar $\mathbf{r}^{21}$ & VASP \\
\hline$v \mathrm{C}=\mathrm{O}$ & 1771 & 1742 & 1784 & 1756 \\
\hline $\mathrm{CH}_{3}(\mathrm{O}) \mathrm{def}$ & $1460 / 1460 / 1440$ & $1448 / 1431 / 1413$ & & \\
\hline $\mathrm{CH}_{3}(\mathrm{C})$ def & $1430 / 1430 / 1375$ & $1425 / 1423 / 1342$ & & \\
\hline$v \mathrm{C}-\mathrm{O}$ & 1248 & 1218 & & \\
\hline$\gamma \mathrm{CH}_{3}(\mathrm{O})$ & $1187 / 1159$ & $1161 / 1130$ & & \\
\hline $\mathrm{vO}-\mathrm{CH}_{3}$ & 1060 & 1033 & & \\
\hline$\gamma \mathrm{CH}_{3}(\mathrm{C})$ & $1036 / 980$ & $1020 / 956$ & & \\
\hline$v \mathrm{C}-\mathrm{C}$ & 844 & $825^{(\mathrm{b})}$ & & \\
\hline$\delta \mathrm{C}=\mathrm{O}_{\text {ip }}$ & 639 & $623^{(b)}$ & & \\
\hline$\delta \mathrm{C}=\mathrm{O}_{\mathrm{op}}$ & 607 & 585 & & \\
\hline CCO def & 429 & 410 & & \\
\hline COC def & 303 & 267 & & \\
\hline$\tau \mathrm{C}-\mathrm{O}$ & 187 & 193 & & \\
\hline$\tau \mathrm{CC}$ & 136 & 75 & & \\
\hline$\tau \mathrm{OCH}_{3}$ & 110 & 149 & & \\
\hline
\end{tabular}

(a) mode assignment according to Ref. ${ }^{12}$ ( $v$ : stretch, $\delta$ : bending, $\tau$ : torsion, $\gamma$ : rocking, def: deformation)

(b) both modes have contributions from $v \mathrm{C}-\mathrm{C}$ and $\delta \mathrm{C}=\mathrm{O}_{\mathrm{ip}}$ 


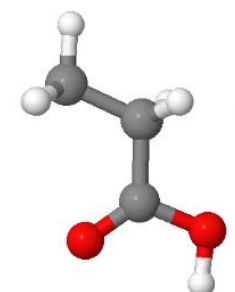

$\operatorname{trans}(\mathrm{t})-\mathrm{PA}$

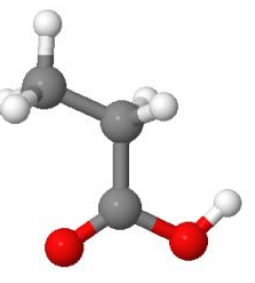

$\operatorname{cis}(\mathrm{t})-\mathrm{PA}$

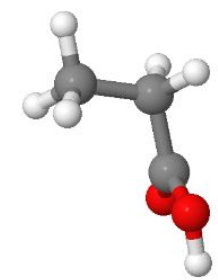

$\operatorname{trans}(\mathrm{g})-\mathrm{PA}$

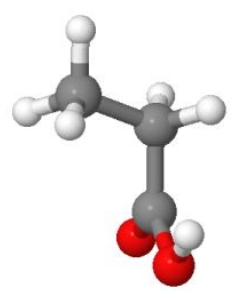

$\operatorname{cis}(\mathrm{g})-\mathrm{PA}$ experiment: $\Delta \mathrm{E}_{\mathrm{t}(\mathrm{t}) \mathrm{t}(\mathrm{g})}=0.04 \mathrm{eV}^{22}$ theory (VASP, this work):

$\Delta \mathrm{E}_{\mathrm{t}(\mathrm{t}) \mathrm{c}(\mathrm{t})}=0.20 \mathrm{eV}, \Delta \mathrm{E}_{\mathrm{t}(\mathrm{t}) \mathrm{t}(\mathrm{g})}=0.06 \mathrm{eV}$, $\Delta \mathrm{E}_{\mathrm{t}(\mathrm{t}) \mathrm{c}(\mathrm{g})}=0.26 \mathrm{eV}$

theory (other work):

$\Delta \mathrm{E}_{\mathrm{t}(\mathrm{t}) \mathrm{c}(\mathrm{t})}=0.26 \mathrm{eV},{ }^{23} 0.22 / 0.23 \mathrm{eV},{ }^{24}$ $0.21 \mathrm{eV}^{25}$

$\Delta \mathrm{E}_{\mathrm{t}(\mathrm{t}) \mathrm{c}(\mathrm{g})}=0.27 \mathrm{eV}^{25}$

Table S9: Calculated geometric parameters for different conformers of free propionic acid in comparison with experimental values. All values are given in $\AA$, except otherwise specified.

\begin{tabular}{|c|c|c|c|c|c|c|}
\hline & \multicolumn{3}{|c|}{$\operatorname{trans}(t)-P A$} & \multirow{2}{*}{$\frac{\operatorname{cis}(\mathbf{t})-\mathbf{P A}^{(\mathrm{a})}}{\text { VASP }}$} & \multirow{2}{*}{$\begin{array}{c}\operatorname{trans}(\mathbf{g})-\mathbf{P A}^{(\mathrm{a})} \\
\text { VASP }\end{array}$} & \multirow{2}{*}{$\frac{\operatorname{cis}(\mathbf{g})-\mathbf{P A}^{(\mathrm{a})}}{\text { VASP }}$} \\
\hline & VASP & Derissen $^{26}$ & Stiefvater $^{27}$ & & & \\
\hline $\mathrm{C}=\mathrm{O}$ & 1.220 & $1.211 \pm 0.003$ & $1.210 \pm 0.010$ & 1.213 & 1.220 & 1.213 \\
\hline $\mathrm{C}-\mathrm{O}$ & 1.369 & $1.367 \pm 0.004$ & $1.352 \pm 0.002$ & 1.375 & 1.370 & 1.376 \\
\hline $\mathrm{C}_{1}-\mathrm{C}_{2}$ & 1.509 & $1.518 \pm 0.010$ & $1.509 \pm 0.002$ & 1.520 & 1.509 & 1.519 \\
\hline $\mathrm{C}_{2}-\mathrm{C}_{3}$ & 1.523 & $1.543 \pm 0.010$ & $1.523 \pm 0.003$ & 1.524 & 1.537 & 1.537 \\
\hline $\mathrm{C}-\mathrm{H}\left(\mathrm{CH}_{2}\right)$ & 1.103 & $1.125 \pm 0.012$ & $1.098 \pm 0.002$ & 1.105 & $1.100 / 1.098$ & $1.104 / 1.098$ \\
\hline $\mathrm{C}-\mathrm{H}\left(\mathrm{CH}_{3}\right)$ & 1.099 & & $\begin{array}{l}1.087 \pm 0.002 \\
1.088 \pm 0.002\end{array}$ & $1.099 / 1.098$ & 1.099 & $1.099 / 1.100$ \\
\hline $\mathrm{O}-\mathrm{H}$ & 0.981 & 0.97 & $0.970 \pm 0.001$ & 0.977 & 0.981 & 0.977 \\
\hline $\mathrm{OCO}$ & $122.2^{\circ}$ & $122.1^{\circ}$ & & $119.4^{\circ}$ & $122.1^{\circ}$ & $119.3^{\circ}$ \\
\hline $\mathrm{CC}-\mathrm{O}$ & $111.5^{\circ}$ & $111.2^{\circ} \pm 0 . .^{\circ} 8$ & $111.2^{\circ} \pm 0.8^{\circ}$ & $115.3^{\circ}$ & $111.8^{\circ}$ & $115.8^{\circ}$ \\
\hline $\mathrm{CC}=\mathrm{O}$ & $126.3^{\circ}$ & $126.7^{\circ} \pm 0.8^{\circ}$ & $126.7^{\circ} \pm 0.8^{\circ}$ & $125.3^{\circ}$ & $126.1^{\circ}$ & $124.8^{\circ}$ \\
\hline $\mathrm{COH}$ & $106.0^{\circ}$ & $107.0^{\circ}$ & $105.8^{\circ} \pm 0.2^{\circ}$ & $109.8^{\circ}$ & $106.0^{\circ}$ & $109.9^{\circ}$ \\
\hline $\mathrm{CCC}$ & $113.3^{\circ}$ & $112.8^{\circ} \pm 1.0^{\circ}$ & $112.7^{\circ} \pm 0.1^{\circ}$ & $113.3^{\circ}$ & $111.5^{\circ}$ & $111.9^{\circ}$ \\
\hline
\end{tabular}

(a) no experimental data available 
Table S10: Calculated vibrational frequencies of different conformers of propionic acid in comparison with experimental values.

\begin{tabular}{|c|c|c|c|c|c|c|c|c|}
\hline & \multicolumn{2}{|c|}{$\operatorname{trans}(\mathbf{t})-\mathbf{P A}$} & \multicolumn{2}{|c|}{$\operatorname{cis}(\mathbf{t})-\mathbf{P A}$} & \multicolumn{2}{|c|}{$\operatorname{trans}(g)-P A$} & \multicolumn{2}{|c|}{$\operatorname{cis}(\mathrm{g})-\mathbf{P A}$} \\
\hline Mode $^{(a)}$ & Solid Ar $\mathbf{A r}^{25}$ & VASP & $\begin{array}{c}\text { Solid } \\
\mathbf{A r}^{25} \\
\end{array}$ & VASP & $\begin{array}{c}\text { Solid } \\
\mathbf{A r}^{25} \\
\end{array}$ & VASP & $\begin{array}{c}\text { Solid } \\
\mathbf{A r}^{25} \\
\end{array}$ & VASP \\
\hline$v \mathrm{C}=\mathrm{O}$ & $1817 / 1776 / 1775$ & 1757 & 1785 & 1792 & 1801 & 1756 & 1802 & 1788 \\
\hline$\delta \mathrm{HCH}_{2}$ & $1466 / 1458$ & $1456 / 1447$ & & & & & & \\
\hline$\delta \mathrm{CH}_{2}$ & $1427 / 1423$ & 1406 & & & & & & \\
\hline $\mathrm{\delta CH}_{3}$ & 1381 & 1371 & & & & & & \\
\hline$\omega \mathrm{CH}_{2}$ & 1378 & 1353 & & & & & & \\
\hline$\delta \mathrm{COH}+\omega \mathrm{CH}_{2}$ & 1224 & 1257 & & & & & & \\
\hline $\mathrm{twCH}_{2}+\gamma \mathrm{CH} 3$ & 1208 & 1241 & & & & & & \\
\hline$v \mathrm{CO}+\delta \mathrm{COH}$ & 1139 & 1117 & & & & & & \\
\hline$\gamma \mathrm{CH}_{3}+\gamma \mathrm{CH}_{2}$ & $1076 / 805$ & $1067 / 787$ & & & & & & \\
\hline$\omega \mathrm{CH}_{3}+v \mathrm{C}-\mathrm{CH}_{3}$ & 1067 & 1051 & & & & & & \\
\hline$v \mathrm{C}-\mathrm{CO}$ & $814 / 812$ & 796 & & & & & & \\
\hline$\tau \mathrm{CO}$ & 626 & 631 & & & & & & \\
\hline$\delta \mathrm{OCO}$ & 608 & 590 & & & & & & \\
\hline$\gamma \mathrm{C}=\mathrm{O}$ & $511 / 503$ & 498 & & & & & & \\
\hline
\end{tabular}

(a) mode assignment according to Ref. ${ }^{25}$ ( $v$ : stretch, $\delta$ : bending, $\tau$ : torsion, $\omega$ : wagging, $\gamma$ : rocking, def: deformation, tw: twisting)

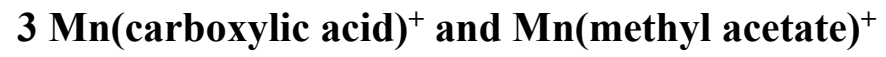

\subsection{Geometric Parameters}

\subsection{1 $\mathrm{Mn}(\mathbf{F A})^{+}$}
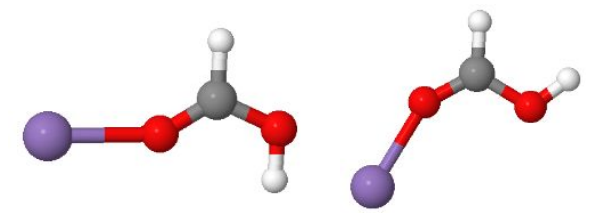

$$
\Delta \mathrm{E}_{\mathrm{tc}}=0.00 \mathrm{eV}
$$

$\mathrm{Mn}(\operatorname{trans}-\mathrm{FA})^{+}$

$\mathrm{Mn}(\text { cis-FA })^{+}$

Table S11: Calculated geometric parameters of $\mathrm{Mn}(\operatorname{trans}-\mathrm{FA})^{+}$and $\mathrm{Mn}(\mathrm{cis}-\mathrm{FA})^{+}$. All values are given in $\AA$, except otherwise specified.

\begin{tabular}{l|l|l|} 
& Mn(trans-FA) & \\
& Mn(cis-FA) $^{+}$ \\
\hline $\mathrm{Mn}-\mathrm{O}$ & 2.084 & 2.087 \\
$\mathrm{C}=\mathrm{O}$ & 1.248 & 1.239 \\
$\mathrm{C}-\mathrm{O}$ & 1.305 & 1.314 \\
$\mathrm{C}-\mathrm{H}$ & 1.101 & 1.104 \\
$\mathrm{O}-\mathrm{H}$ & 0.987 & 0.983 \\
$\mathrm{OCO}$ & $124.1^{\circ}$ & $120.6^{\circ}$ \\
$\mathrm{HC}-\mathrm{O}$ & $112.4^{\circ}$ & $118.5^{\circ}$ \\
$\mathrm{HC}=\mathrm{O}$ & $123.5^{\circ}$ & $120.9^{\circ}$ \\
$\mathrm{COH}$ & $112.0^{\circ}$ & $112.3^{\circ}$
\end{tabular}




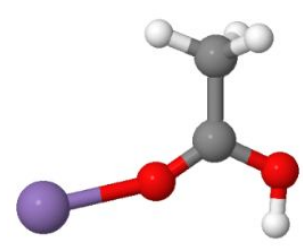

$\mathrm{Mn}(\operatorname{trans}-\mathrm{FA})^{+}$

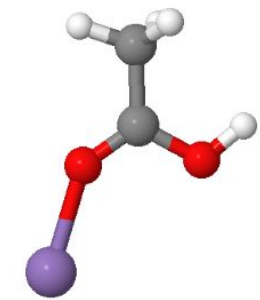

$\mathrm{Mn}(\text { cis-FA })^{+}$

$$
\Delta \mathrm{E}_{\mathrm{tc}}=0.01 \mathrm{eV}
$$

Table S12: Calculated geometric parameters of $\mathrm{Mn}(\operatorname{trans}-\mathrm{FA})^{+}$and $\mathrm{Mn}(\mathrm{cis}-\mathrm{FA})^{+}$. All values are given in $\AA$, except otherwise specified.

\begin{tabular}{l|l|l|} 
& \multicolumn{1}{|c|}{$\operatorname{Mn}(\operatorname{trans}-\mathbf{A A})^{+}$} & \multicolumn{1}{|c|}{${\mathbf{M n}(\mathbf{c i s}-\mathbf{A A})^{+}}^{+}$} \\
\hline $\mathrm{Mn}-\mathrm{O}$ & 2.040 & 2.063 \\
$\mathrm{C}=\mathrm{O}$ & 1.260 & 1.252 \\
$\mathrm{C}-\mathrm{O}$ & 1.318 & 1.328 \\
$\mathrm{C}-\mathrm{C}$ & 1.485 & 1.488 \\
$\mathrm{C}-\mathrm{H}$ & $1.094 / 1.101 / 1.101$ & $1.094 / 1.102 / 1.102$ \\
$\mathrm{O}-\mathrm{H}$ & 0.985 & 0.981 \\
$\mathrm{OCO}$ & $120.3^{\circ}$ & $116.9^{\circ}$ \\
$\mathrm{CC}-\mathrm{O}$ & $114.5^{\circ}$ & $120.2^{\circ}$ \\
$\mathrm{CC}=\mathrm{O}$ & $125.2^{\circ}$ & $122.8^{\circ}$ \\
$\mathrm{COH}$ & $111.1^{\circ}$ & $112.5^{\circ}$
\end{tabular}


3.1.3 $\mathrm{Mn}(\mathrm{MA})^{+}$

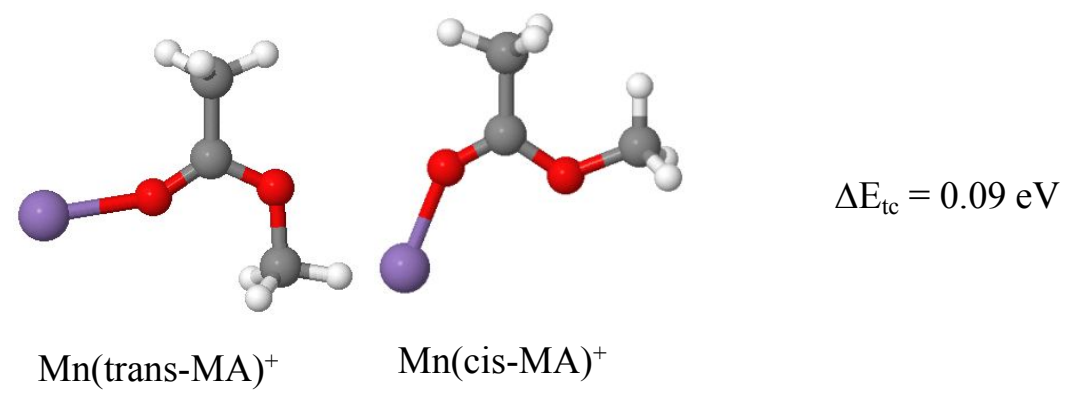

Table S13: Calculated geometric parameters of free methyl acetate in trans- and cis-geometry. All values are given in $\AA$, except otherwise specified.

\begin{tabular}{l|l|l|} 
& Mn(trans-MA) & \multicolumn{1}{|c|}{ Mn(cis-MA) $^{+}$} \\
\hline $\mathrm{Mn}-\mathrm{O}$ & 2.018 & 2.036 \\
$\mathrm{C}=\mathrm{O}$ & 1.266 & 1.262 \\
$\mathrm{C}-\mathrm{O}$ & 1.307 & 1.315 \\
$\mathrm{O}-\mathrm{CH}_{3}$ & 1.464 & 1.462 \\
$\mathrm{C}-\mathrm{C}$ & 1.488 & 1.490 \\
$\mathrm{C}-\mathrm{H}(\mathrm{O})$ & $1.096 / 1.097 / 1.093$ & $1.096 / 1.096 / 1.094$ \\
$\mathrm{C}-\mathrm{H}(\mathrm{C})$ & $1.103 / 1.095 / 1.098$ & $1.101 / 1.101 / 1.094$ \\
$\mathrm{OCO}$ & $121.2^{\circ}$ & $116.3^{\circ}$ \\
$\mathrm{CC}-\mathrm{O}$ & $115.1^{\circ}$ & $122.9^{\circ}$ \\
$\mathrm{CC}=\mathrm{O}$ & $123.7^{\circ}$ & $120.8^{\circ}$ \\
$\mathrm{COC}$ & $119.4^{\circ}$ & $123.5^{\circ}$
\end{tabular}




\subsection{4 $\mathrm{Mn}(\mathbf{P A})^{+}$}
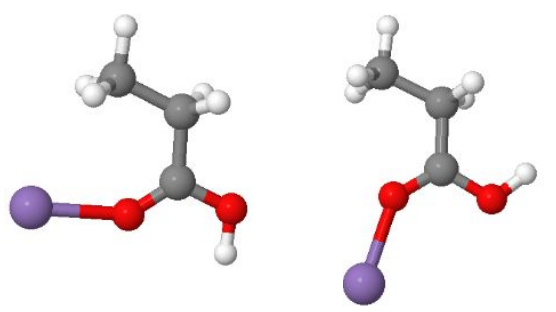

$\operatorname{Mn}(\operatorname{trans}(\mathrm{t})-\mathrm{PA})^{+}$
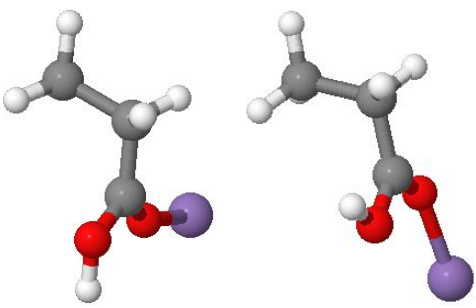

$$
\begin{aligned}
& \Delta \mathrm{E}_{\mathrm{t}(\mathrm{t}) \mathrm{c}(\mathrm{t})}=0.04 \mathrm{eV} \\
& \Delta \mathrm{E}_{\mathrm{t}(\mathrm{t}) \mathrm{t}(\mathrm{g})}=0.08 \mathrm{eV} \\
& \Delta \mathrm{E}_{\mathrm{t}(\mathrm{t}) \mathrm{c}(\mathrm{g})}=0.08 \mathrm{eV}
\end{aligned}
$$

Table S14: Calculated geometric parameters for different conformers of free propionic acid in comparison with experimental values. All values are given in $\AA$, except otherwise specified.

\begin{tabular}{l|l|l|l|l|} 
& Mn(trans(t)-PA $)^{+}$ & Mn(cis(t)-PA $)^{+}$ & Mn(trans(g)-PA $)^{+}$ & $\operatorname{Mn}(\operatorname{cis}(\mathrm{g})-\mathrm{PA})^{+}$ \\
\hline $\mathrm{Mn}-\mathrm{O}$ & 2.053 & 2.059 & 2.033 & 2.057 \\
$\mathrm{C}=\mathrm{O}$ & 1.259 & 1.253 & 1.263 & 1.254 \\
$\mathrm{C}-\mathrm{O}$ & 1.318 & 1.330 & 1.318 & 1.329 \\
$\mathrm{C}_{1}-\mathrm{C}_{2}$ & 1.494 & 1.496 & 1.489 & 1.493 \\
$\mathrm{C}_{2}-\mathrm{C}_{3}$ & 1.522 & 1.524 & 1.547 & 1.547 \\
$\mathrm{C}-\mathrm{H}\left(\mathrm{CH}_{2}\right)$ & $1.104 / 1.104$ & $1.107 / 1.106$ & $1.100 / 1.099$ & $1.102 / 1.098$ \\
$\mathrm{C}-\mathrm{H}\left(\mathrm{CH}_{3}\right)$ & $1.098 / 1.103 / 1.103$ & $1.097 / 1.098 / 1.098$ & $1.097 / 1.099 / 1.098$ & $1.097 / 1.098 / 1.099$ \\
$\mathrm{O}-\mathrm{H}$ & 0.985 & 0.981 & 0.985 & 0.981 \\
$\mathrm{OCO}$ & $120.4^{\circ}$ & $116.8^{\circ}$ & $120.1^{\circ}$ & $116.8^{\circ}$ \\
$\mathrm{CC}-\mathrm{O}$ & $114.7^{\circ}$ & $119.9^{\circ}$ & $115.0^{\circ}$ & $120.7^{\circ}$ \\
$\mathrm{CC}=\mathrm{O}$ & $124.9^{\circ}$ & $123.2^{\circ}$ & $124.9^{\circ}$ & $122.5^{\circ}$ \\
$\mathrm{COH}$ & $110.9^{\circ}$ & $112.2^{\circ}$ & $111.1^{\circ}$ & $112.1^{\circ}$ \\
$\mathrm{CCC}$ & $114.8^{\circ}$ & $115.1^{\circ}$ & $110.5^{\circ}$ & $110.6^{\circ}$
\end{tabular}

\section{Bader Charge Analysis}
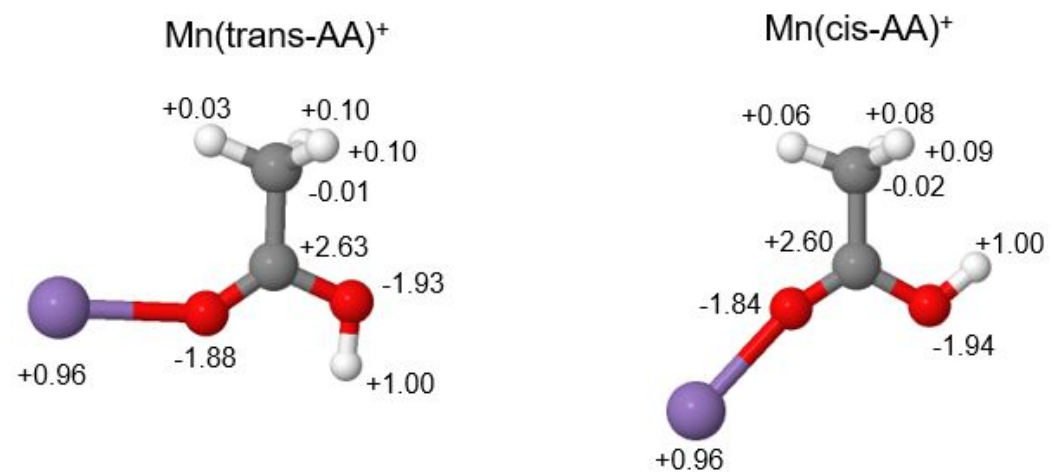

Figure S1: Bader charges ${ }^{28-30}$ for Mn(trans-AA $)^{+}$and $\mathrm{Mn}(\text { cis-AA })^{+}$. 
1. Kresse, G.; Hafner, J. Ab. Initio molecular dynamics for liquid metals. Phys. Rev. B 1993, 47, 558-561.

2. Kresse, G.; Hafner, J. Ab initio molecular-dynamics simulation of the liquid-metal amorphous-semiconductor transition in germanium. Phys. Rev. B 1994, 49, 14251-14269.

3. Kresse, G.; Furthmüller, J. Efficient iterative schemes for ab initio total-energy calculations using a plane-wave basis set. Phys. Rev. B 1996, 54, 11169-11185.

4. Kresse, G.; Furthmüller, J. Efficiency of ab-initio total energy calculations for metals and semiconductors using a plane-wave basis set. Comp. Mat. Sci. 1996, 6, 15-50.

5. Barnett, R. N.; Landman, U. Born-oppenheimer molecular-dynamics simulations of finite systems: Structure and dynamics of $\left(\mathrm{H}_{2} \mathrm{O}\right)_{2}$. Phys. Rev. B 1993, 48, 2081-2097.

6. Hocking, W. H. The other rotamer of formic acid, cis-hcooh. Z. Naturf. A 1976, 31, 1113-1121.

7. Kwei, G. H.; Curl Jr., R. F. Microwave spectrum of $\mathrm{O}^{18}$ formic acid and structure of formic acid. J. Chem. Phys. 1960, 32, 1592-1594.

8. Schomaker, V.; O'Gorman, J. M. An electron diffraction investigation of formic acid monomer. J. Am. Chem. Soc. 1947, 69, 2638-2644.

9. Karle, I. L.; Karle, J. Structure of the monomer of formic acid. J. Chem. Phys. 1954, 22, 43-45.

10. Lerner, R. G.; Dailey, B. P.; Friend, J. P. Microwave spectrum and structure of formic acid. J. Chem. Phys. 1957, 26, 680-683.

11. Bjarnov, E.; Hocking, W. H. The structure of the other rotamer of formic acid, cis-HCOOH. Z. Naturf. A 1978, 33, 610.

12. Shimanouchi, T., Tables of molecular vibrational frequencies consolidated volume I. National Bureau of Standards: 1972.

13. Maçôas, E. M. S.; Lundell, J.; Pettersson, M.; Khriachtchev, L.; Fausto, R.; Räsänen, M. Vibrational spectroscopy of cis- and trans-formic acid in solid argon. J. Mol. Spectrosc. 2003, 219, 7080.

14. Sato, H.; Hirata, F. The syn-/anti-conformational equilibrium of acetic acid in water studied by the RISM-SCF/MCSCF method. J. Mol. Struct.: THEOCHEM 1999, 461-462, 113-120.

15. Gao, Q.; Leung, K. T. Hydrogen-bonding interactions in acetic acid monohydrates and dihydrates by density-functional theory calculations. J. Chem. Phys. 2005, 123, 074325.

16. Terhorst, J. P.; Jorgensen, W. L. E/Z energetics for molecular modeling and design. J. Chem. Theory Comput. 2010, 6, 2762-2769.

17. Senent, M. L. Ab initio determination of the torsional spectra of acetic acid. Mol. Phys. 2001, 99, 1311-1321.

18. Johnson III , R. D., NIST computational chemistry comparison and benchmark database, NIST standard reference database number 101 http://cccbdb.nist.gov/, 2018.

19. Derissen, J. L. A reinvestigation of the molecular structure of acetic acid monomer and dimer by gas electron diffraction. J. Mol. Struct. 1971, 7, 67-80.

20. Maçôas, E. M. S.; Khriachtchev, L.; Pettersson, M.; Fausto, R.; Räsänen, M. Rotational isomerism in acetic acid: The first experimental observation of the high-energy conformer. J. Am. Chem. Soc. 2003, 125, 16188-16189.

21. Blom, C. E.; Günthard, H. H. Rotational isomerism in methyl formate and methyl acetate; a low-temperature matrix infrared study using thermal molecular beams. Chem. Phys. Lett. 1981, 84, 267-271.

22. Wiberg, K. B.; Laidig, K. E. Acidity of (z)- and (e)-methyl acetates: Relationship to Meldrum's acid. J. Am. Chem. Soc. 1988, 110, 1872-1874.

23. Teixeira-Dias, J. J. C.; Fausto, R. A molecular mechanics force field for conformational analysis of simple acyl chlorides, carboxylic acids and esters. J. Mol. Struct. 1986, 144, 199-213.

24. Lii, J.-H. Molecular mechanics (MM4) studies of carboxylic acids, esters, and lactones. J. Phys. Chem. A 2002, 106, 8667-8679.

25. Maçôas, E. M. S.; Khriachtchev, L.; Pettersson, M.; Fausto, R.; Räsänen, M. Internal rotation in propionic acid: Near-infrared-induced isomerization in solid argon. J. Phys. Chem. A 2005, 109, 36173625. 
26. Derissen, J. L. An investigation of the structure of propionic acid monomer and dimer by gas electron diffraction. J. Mol. Struct. 1971, 7, 81-88.

27. Stiefvater, O. L. Microwave spectrum of propionic acid. II. Structure of cis-propionic acid by double resonance modulation microwave spectroscopy. J. Chem. Phys. 1975, 62, 244-256.

28. Yu, M.; Trinkle, D. R. Accurate and efficient algorithm for Bader charge integration. J. Chem. Phys. 2011, 134, 064111.

29. Sanville, E.; Kenny, S. D.; Smith, R.; Henkelman, G. Improved grid-based algorithm for Bader charge allocation. J. Comput. Chem. 2007, 28, 899-908.

30. Tang, W.; Sanville, E.; Henkelman, G. A grid-based Bader analysis algorithm without lattice bias. J. Phys.: Cond. Mat. 2009, 21, 084204. 